\title{
THE LINEAR POLARIZATION OF EXTRAGALACTIC RADIO SOURCES AT MILLIARCSECOND RESOLUTION
}

\author{
J. F. C. Wardle and D. H. Roberts \\ Department of Physics \\ Brandeis University \\ Waltham, MA 02254 USA
}

\begin{abstract}
We review the polarization sensitive VLBI observations of compact extragalactic radio sources that have been made to date, and discuss some preliminary physical results suggested by the data.
\end{abstract}

\section{INTRODUCTION}

Maps of the distribution of linear polarization in non-thermal radio sources can give a wealth of information that is not available from total intensity maps. This includes the topology of the magnetic field, the distribution of Faraday rotating material both inside and surrounding the radio emitting region, and the low-energy end of the relativistic electron spectrum. Also, relativistic and kinematic effects can impose distinctive signatures on the observed polarization. This information can allow important physical parameters to be measured and can help discriminate among theoretical models of radio sources.

The first map of the distribution of linear polarization in a compact radio source at VLBI resolution was of the quasar $3 \mathrm{C} 454.3$ at $\lambda 11.1 \mathrm{~cm}$, published by Cotton et al. (1984). Since then, the group at Brandeis University has observed over 40 sources, some at as many as six epochs (Roberts et al. 1984; Wardle et al. 1986; Wardle and Roberts 1986a,b; Roberts and Wardle 1986, 1987; Roberts, Gabuzda and Wardle 1987; several papers, this symposium). Similar observational programs have also been initiated by several groups using the European VLBI network, but no results are available yet. Making polarization maps at VLBI resolution is not without its technical problems, but we shall not discuss them here (see Roberts et al. 1984, and in preparation).

In section 2, we summarize the results obtained to date. Eight sources are discussed in more detail in section 3 . In sections 4 and 5 we attempt to draw some overall conclusions from these data; section 4 is concerned with the configuration of the magnetic field inferred from the radio (and optical) polarization data, while section 5 is concerned with the distribution of Faraday rotating material inside and surrounding the radio sources.

\section{SUMMARY OF OBSERVATIONAL RESULTS}

Table I summarizes twelve VLBI polarization observations on eight sources, and includes all results available to date. Column 1 gives the common name of each source, column 2 lists the epochs of observation, and column 3 gives the optical identification (' $B$ ' $=B L$ Lac Object, ' $\mathrm{Q}$ '= quasar, ' $\mathrm{G}$ '= galaxy). Columns 4,5 and 6 list the flux density, degree of 
polarization, and position angle (of the electric vector) for the 'core' component. Columns 7,8 and 9 give the same quantities for the 'jet' component. Where the jet consists of more than one component, parameters are given for the component with the largest polarized flux density. Column 10 gives the position angle of elongation of the VLBI structure. The polarization position angles are uncorrected for Faraday rotation. This has not yet been measured directly at VLBI resolution, but for the 'jet' components it is probably small in most cases (e.g., Rudnick and Jones 1983), though BL Lac itself is certainly an exception $\left(\mathrm{RM}=-198 \mathrm{rad} \mathrm{m}^{-2}\right.$, Rudnick et al. 1984). All observations were made at a wavelength of $6 \mathrm{~cm}$, except 3C454.3 $(\lambda 11.1 \mathrm{~cm})$.

Table I: Summary of VLBI Polarization Observations

\begin{tabular}{|c|c|c|c|c|c|c|c|c|c|}
\hline$\overline{\text { Source }}$ & Epoch & ID & $\mathbf{s ( j y )}$ & $\begin{array}{l}\text { Core } \\
\mathrm{m}(\%)\end{array}$ & $\begin{array}{l}\ldots . \\
\chi(\operatorname{deg})\end{array}$ & $\mathrm{S}(\mathrm{Jy})$ & $\begin{array}{l}\text {.Jet } . \\
\text { m(\%) }\end{array}$ & $\begin{array}{l}\ldots \\
\chi(\operatorname{deg})\end{array}$ & $\begin{array}{c}\text { VLBI PA } \\
\theta \text { (deg) }\end{array}$ \\
\hline $0235+164$ & $\begin{array}{l}1981.9 \\
1982.9\end{array}$ & B & $\begin{array}{l}2.1 \\
1.7\end{array}$ & $\begin{array}{l}1.4 \\
1.2\end{array}$ & $\begin{array}{c}39 \\
110\end{array}$ & - & - & $\begin{array}{l}- \\
-\end{array}$ & - \\
\hline $0735+178$ & 1982.9 & B & 1.3 & 4.1 & 5 & 0.2 & 8.3 & 46 & 72 \\
\hline OJ287 & $\begin{array}{l}1981.9 \\
1982.9\end{array}$ & B & $\begin{array}{l}2.9 \\
2.8\end{array}$ & $\begin{array}{l}3.4 \\
4.6\end{array}$ & $\begin{array}{c}160 \\
99\end{array}$ & $\begin{array}{l}0.3 \\
0.4\end{array}$ & $\begin{array}{l}64 . \\
23 .\end{array}$ & $\begin{array}{l}83 \\
78\end{array}$ & -116 \\
\hline $1749+096$ & 1981.9 & B & 1.8 & 6.5 & 39 & - & $>40$ & -68 & 13 \\
\hline BL Lac & 1984.2 & B & 0.8 & 2.8 & -68 & 1.2 & 7.4 & $-35^{a}$ & 10 \\
\hline $3 \mathrm{C} 120$ & 1982.9 & G & 1.0 & $<0.7$ & - & 0.2 & 11. & 22 & -96 \\
\hline $3 \mathrm{C} 345$ & $\begin{array}{l}1981.9 \\
1983.9 \\
1984.2\end{array}$ & $\mathbf{Q}$ & $\begin{array}{l}6.4 \\
9.0 \\
8.6\end{array}$ & $\begin{array}{c}<1.0 \\
1.6 \\
2.0\end{array}$ & $\begin{array}{c}- \\
-2 \\
-27\end{array}$ & $\begin{array}{l}1.8 \\
1.2 \\
1.0\end{array}$ & $\begin{array}{l}11 . \\
11 . \\
12 .\end{array}$ & $\begin{array}{l}22 \\
30 \\
28\end{array}$ & -82 \\
\hline $3 \mathrm{C} 454.3^{b}$ & 1980.6 & $\mathbf{Q}$ & - & - & - & - & 20. & - & 12. \\
\hline
\end{tabular}

\section{DESCRIPTION OF INDIVIDUAL SOURCES}

0235+164: This is a well known violently variable $B L$ Lac object, that exhibits two absorption line redshifts $\left(z_{a}=0.524,0.852\right)$, and shows variable HI absorption at the lower redshift (Wolfe et al. 1982). Cohen et al. (1986) have recently determined an emission redshift $\left(z_{e}=0.94\right)$. The radio polarization exhibited a position angle that rotated linearly with time during a large outburst in 1975 (Ledden and Aller 1978); this was interpreted by Blandford and Königl (1979) as changing relativistic aberration. Its VLBI structure is very compact at all wavelengths. Båath (1984) found faint extensions to the north-east at three epochs, but at position angles that varied between $0^{\circ}$ and $45^{\circ}$. We also find indications of structure to the north-east, but our dynamic range and resolution are insufficient to determine its nature.

0735+178: This BL Lac object has been called a 'cosmic conspirator' because several self-absorbed radio components 'conspire' to form a remarkably flat overall spectrum 
(Cotton et al. 1980). Its optical spectrum shows a single absorption redshift $\left(z_{a}=0.424\right.$; Carswell et al. 1974). Baaath (1984) found the VLBI structure to consist of a bright core and a knot approximately 5 mas to the north-east, with evidence of a weaker superluminal component between these. Our observations (Wardle and Roberts 1986; Gabuzda et al. this symposium) show the two stronger components, together with a component located midway between them; it is unclear whether the latter is the superluminal component seen by Bååth. The inner knot is weakly polarized $\left(m \simeq 3 \%\right.$ at $\left.\chi \simeq 20^{\circ}\right)$. The polarization of the outer knot is $m \simeq 8 \%$ at position angle $\chi \simeq 45^{\circ}$. The rotation measure for $0735+178$ is given as $-304 \mathrm{rad} \mathrm{m}^{-2}$ by Simard-Normandin et al. (1981). This value may have been affected by the source variability, since long-term monitoring programs indicate a much more modest value (Altschuler and Wardle 1976; Aller et al. 1985). If Faraday rotation is not important, then the magnetic field in the outer knot is roughly perpendicular to the jet.

OJ287: This is an extremely active BL Lac object at $z_{e}=0.306$ (Sitko and Junkkarinen 1985). The details of the remarkable VLBI polarization structure are given in Roberts et al. (1987, and this symposium). At each epoch, the structure can be modeled as a core and two knots forming a jet at position angle $\theta \simeq-115^{\circ}$. Over a year, both knots increased their separation from the core by about 0.3 mas, corresponding to an apparent superluminal velocity of about $3.3 h^{-1} \mathrm{c}$ for each knot, assuming $q_{0}=0.5$ and a Hubble constant of $100 \mathrm{~h} \mathrm{~km} / \mathrm{s} \cdot \mathrm{Mpc}$. The polarizations of the core and the (brighter) inner knot also changed markedly between the two epochs - the position angle of the core rotated by $61^{\circ}$, and the degree of polarization of the inner knot dropped from $64 \%$ to $23 \%$. The rotation measure for $0 J 287$ is quite uncertain because the source is so variable. If Faraday rotation is not important, then the magnetic field in the knots is roughly perpendicular to the direction of the jet.

1749+096: This a BL Lac object for which no redshift is available. The polarized structure consists of a comparatively'strongly polarized component coincident with the bright core, and a 'knot' separated by about 0.8 mas at position angle $\theta \simeq 13^{\circ}$ (Gabuzda et al. this symposium). Our observations were of insufficient dynamic range to detect this ' $k$ not' in total intensity, but the degree of polarization of the knot must be in excess of $40 \%$ $\left(\chi \simeq 112^{\circ}\right)$. The integrated rotation measure of this source is given by Rudnick and Jones (1983) as $105 \mathrm{rad} \mathrm{m}^{-2}$. If this applies to the knot, the magnetic field is roughly parallel to the direction of the 'jet'.

BL Lac: Our results for the prototype BL Lac object itself $\left(z_{\text {gal }}=\mathbf{0 . 0 6 8 8}\right.$; Miller et al. 1978) are preliminary (see Gabuzda et al. this symposium). Total intensity maps at $\lambda 2.8 \mathrm{~cm}$ by Mutel and Phillips (1984, and this symposium) show a complex, elongated, and curved structure, at position angle $\theta \simeq-170^{\circ}$. The core is probably comparatively weak and located north of the brightest knot. The knots in the jet exhibit superluminal motion at $2.4 h^{-1}$ c. The bright northern knot is unresolved on transatlantic baselines (in both cross-hand and parallel hand fringes) and appears to be the component associated with the strongly polarized 1983 outburst (Aller, Aller, and Hughes 1985). Hughes, Aller, and Aller (1985) have convincingly modeled this outburst as a region of compression driven by a hot 'piston' traveling along the jet. Our observations were made at the end of this outburst. The rotation measure of BL Lac is large $\left(-198 \mathrm{rad} \mathrm{m}^{-2}\right.$; Rudnick et al. 1984); assuming this applies to the strongest polarized component, then the magnetic field is transverse to the jet in that knot, as required by the model of Hughes et al..

3C120: This galaxy provides the nearest $\left(z_{\text {gal }}=0.033\right)$ example of superluminal motion. The source contains a complex jet which exhibits structure on scales from milliarcseconds 
to arcminutes (Walker et al. 1987). The VLBI polarization map (Roberts and Wardle 1986) detected only the innermost knot of the jet, and most of the polarized flux was heavily resolved. No polarized emission was detected from the bright core, and its degree of polarization is certainly less than $1 \%$. The integrated rotation measure is $-3 \mathrm{rad} \mathrm{m}^{-2}$ (Simard-Normandin et al. 1981). If this applies to the detected knot, then its magnetic field is roughly perpendicular to the direction of the jet.

3C345: This well known superluminal quasar $\left(z_{e}=0.595\right)$ has been extensively observed with total intensity VLBI by the Caltech group (e.g., Cohen et al. 1983; Biretta et al. 1986), and consists of a core and several bright knots in a curved jet with an average position angle $-75^{\circ}$. The details of the polarization structure are given in Wardle et al. (1986, and this symposium). Each knot is detected in polarized emission as it emerges from the core, while the core itself appears to remain essentially unpolarized. The position angles of polarization of the four detected knots are all different, though the knot with the largest polarized flux density (C3) is polarized nearly perpendicular to the jet. If the integrated rotation measure $\left(23 \mathrm{rad} \mathrm{m}^{-2}\right.$; Simard-Normandin et al. 1981) applies to this component, then the magnetic field in $\mathrm{C} 3$ is nearly parallel to the jet. In the other components, either the field is not parallel to the jet or there is a large gradient of rotation measure along the jet.

3C454.3: This quasar $\left(z_{e}=0.859\right)$ has been less extensively observed at VLBI resolution than $3 \mathrm{C} 345$, but certainly has a core-jet structure with evidence of superluminal motion at $v \sim 10 h^{-1}$ c (Pauliny-Toth et al. 1984). Great technical problems had to be overcome in order for Cotton et al. (1984) to make their polarization map at $\lambda 11.1 \mathrm{~cm}$. They found that the position angle of polarization varied continuously down the jet. This, together with a rotation measure of $-57 \mathrm{rad} \mathrm{m}^{-2}$ (Simard-Normandin et al. 1981), makes it difficult to determine the orientation of the magnetic field. Long-term monitoring of the integrated polarization of this source (Altschuler and Wardle 1976; Aller et al. 1985) show a preferred (intrinsic) position angle of polarization of 0 to $10^{\circ}$. This would imply a magnetic field aligned roughly along the jet, but it is not clear if this refers to the core or to the jet itself.

\section{THE STRUCTURE OF THE MAGNETIC FIELD}

The first point to be made from the data presented is that although the integrated polarizations of compact radio sources are typically quite modest (usually $\leq 5 \%$, e.g. Altschuler and Wardle 1977; Aller et al. 1985), individual VLBI components can be very strongly polarized indeed. In $0 J 287$, the degree of polarization approaches the theoretical limit for synchrotron radiation from a power-law electron energy distribution, implying an extremely well-ordered magnetic field. In general, much of the polarized flux comes from the optically thin 'jet' components, and the degree of polarization of the cores is always lower than that of the jets, and is sometimes $<1 \%(3 \mathrm{C} 120,3 \mathrm{C} 345)$. Because of this, polarization maps can reveal extended structure that is not visible on total intensity maps (because of their finite dynamic range). The best example of this is $0 J 287$ (Roberts et al. 1987, and this symposium). Total intensity observations show an extremely compact structure, and the source is frequently used as a calibrator. In our first epoch observations, the polarized structure is that of a well-resolved, almost equal double. Moreover, the polarized components appear to be separating at $3.3 h^{-1}$ c. None of this detail is apparent in total intensity maps made so far.

From Table I, we can compare the orientation of the projected magnetic field in the strongest jet component to the orientation of the VLBI jet itself. It is necessary to assume 
either that the Faraday rotation is not large, or that the integrated rotation measure applies to the VLBI component under consideration. While these assumptions usually are true (Rudnick and Jones 1983), we shall argue below that they are not always so. With this caveat in mind, four sources have a projected magnetic field roughly perpendicular to the direction of their jet $(0735+178, \mathrm{OJ} 287$, BL Lac, $3 \mathrm{C} 120)$, and three sources have a projected magnetic field roughly parallel to the direction of their jet $(1749+096,3 \mathrm{C} 345$, and possibly 3C454.3).

These alignments are very rough, however, and many more observations are required to determine the true magnetic field topology and how (and whether) it changes with time. Nevertheless, this mild preference for magnetic fields to be aligned roughly parallel or perpendicular to the orientation of VLBI structure is in accord with the results of Aller et al. (this symposium), Wrobel et al. (this symposium), Rusk and Seaquist (1985), and Rusk (this symposium), based on integrated polarization measurements on a much larger number of sources. (It should be noted that the magnetic field directions inferred from such measurements may not be strictly comparable to those determined from VLBI polarization data, since the former may refer to the VLBI core component, or to structure on a larger scale that is resolved by VLBI). Two of these results seem to be particularly interesting. Aller et al. find several sources (of which BL Lac is one) for which the inferred magnetic field is parallel to the jet during quiescent periods, and perpendicular to the jet during strongly polarized outbursts. They ascribe this behavior to weak shocks in the jet that compress a nearly disordered magnetic field, thus greatly enhancing the transverse field components (see also Hughes, Aller and Aller 1985, and this symposium). Rusk has examined the magnetic field alignment as a function of optical identification. He finds that for galaxies and quasars the inferred magnetic field direction tends to be parallel to the jet, while for BL Lac objects, the distribution of alignments is bimodal, but with the majority of sources exhibiting a magnetic field transverse to the jet. This is an intriguing result that seems to have no obvious explanation within the standard theoretical models for compact radio sources. It might suggest that there is more than one way to form bright emission knots in a jet, or that the knots evolve in different ways, perhaps depending on the local environment (e.g., the presence of gas?). Certainly, future studies of this type should pay attention to whether the radio source is in an active or a quiescent phase, and to any alignment changes during outbursts.

Optical polarization data also suggest certain correlations with the orientation of the VLBI structure which can give information on the magnetic field structure on scales smaller than those accessible to VLBI. 'Low polarization quasars' $\left(m_{\text {opt }}<3 \%\right)$ tend to have the optical polarization position angle parallel to the radio structure (Stockman, Angel, and Miley 1979). In these sources, it is not clear that there is any non-thermal component to the optical continuum emission, and the polarization may be induced by scattering from an accretion disc or an oblate cloud of material aligned perpendicular to the radio axis. 'High polarization quasars' $\left(m_{o p t}>3 \%\right)$ and BL Lac objects, which are often very strongly polarized (sometimes as high as $40 \%$ ), exhibit more complex behavior (Angel and Stockman 1980). These sources are believed to contain a strong component of non-thermal emission. The most variable sources exhibit no preferred position angle for the optical polarization. However, in sources where the range of optical position angles is not too extreme, the direction of the VLBI structure tends to fall within this range (Ulrich 1984; Rusk and Seaquist 1985; Sitko et al. 1985; Smith et al. 1987). Two sources that show this behavior are 3C345 and BL Lac. If the polarized optical emission is synchrotron radiation, then it comes from regions where the magnetic field has a tendency to be transverse to the direction of the jet (assuming that the emission region is optically thin at optical wavelengths). In some sources (e.g., BL Lac; Moore et al. 1982, 1987), the optical polarization behavior 
is consistent with a random walk or 'Christmas tree' model, where the optical emission comes from many short-lived independent regions (size $\gtrsim 1 \mathrm{lt}-\mathrm{hr}$ ), each of high intrinsic polarization. By analogy with the variable radio emission from BL Lac, these too might be shocks, where intense particle acceleration is also taking place. Jones et al. (1985) have discussed a similar picture to explain the integrated radio polarization data.

The optical observations cannot, of course, determine the exact location of the emission, though it is usually assumed that the non-thermal optical emission originates deep inside the radio 'core', close to the central engine. For some sources, the radio and optical polarizations are sometimes well correlated (e.g., 3C345 and BL Lac; Sitko et al. 1985). For both of these sources, we find that most of the polarized radio emission comes from components in the jet. It is therefore possible that part of the polarized optical emission is also associated with these jet components. If changes in the optical polarization are found that correlate with with changes in the radio polarization of a VLBI jet component, then this possibility would have to be taken seriously. Such a result would place extraordinary requirements on the particle acceleration mechanism in the jet components. To explore this possibility, M. Sitko is making optical polarization measurements simultaneously with our VLBI observing runs.

There are, of course, many ways of producing either predominantly parallel or transverse magnetic fields (Begelman, Blandford, and Rees 1984). In an expansion that conserves magnetic flux, $\mathbf{B}_{\perp} \sim r^{-1}$, and $\mathbf{B}_{\|} \sim r^{-2}$, where $r$ is the transverse dimension of the jet. Thus the transverse field components are enhanced relative to the parallel component. This effect has certainly been seen in arcsecond scale jets (Bridle and Perley 1984). A transverse compression or shock has a similar effect: $\mathbf{B}_{\perp} \sim k^{-1}$, and $\mathbf{B}_{\|}$is unchanged, where $k$ is the degree of compression (unit length is compressed to length $k$; Hughes et al. 1985). Mild degrees of compression can produce quite high degrees of polarization when viewed from the correct angle (i.e., nearly transverse to the plane of compression; see also Laing 1980). Note that the 'viewing angle' is determined in the rest frame of the emitting material. For a relativistic jet with a Lorentz factor $\Gamma$, the polarization is a maximum if the jet makes an angle $\sim 1 / \Gamma$ to the line of sight, the same angle that maximizes apparent superluminal motion. Other effects of aberration on the observed polarization are discussed by Blandford and Königl (1979), Björnsson (1982), and Wardle and Roberts (1986a).

A predominantly longitudinal field is inevitably produced by shear due to any velocity gradient between the boundary and the center of the jet. If there is any interaction between the jet and the ambient medium then there will be boundary layer mixing and mass entrainment (De Young 1986), possibly observable as a center-brightened polarization distribution, and eventual deceleration due to momentum transfer. More complicated magnetic field structures have also been proposed. Two of these are the 'transverse self-similar' model of Chan and Henriksen (1980), which pays particular attention to the confinement of the jet, and the 'twisted rope' model of Königl and Choudhari (1985), which is a minimum energy configuration that predicts regular changes in polarization along the jet.

It is possible that all of these mechanisms could operate within the same jet. As the dynamic range of the polarization observations improves, we expect to see a rich variety of magnetic field topologies, and to be able to follow the dynamical evolution of the magnetic field in individual jet components.

\section{THE DISTRIBUTION OF FARADAY ROTATING MATERIAL}

In this section we will discuss how the polarization observations can be used to probe magneto-active plasma inside and in the vicinity of the radio emitting regions. We are particularly interested in why the core components in some sources are so weakly polarized. 
The importance of Faraday rotation is measured by the 'Faraday depth', $\xi=8.1 \times$ $10^{5} \lambda^{2} \int n_{e} B \cdot d l$, where $\lambda(\mathrm{m})$ is the observing wavelength in the rest frame of the Faraday rotating plasma; $n_{e}\left(\mathrm{~cm}^{-3}\right)$ is the density of thermal electrons; $\mathbf{B}$ (gauss) is the magnetic field; and $\mathrm{dl}(\mathrm{pc})$ is an element of length along the line of sight. In general the integration extends from the back of the source to the observer. If there are reversals of the magnetic field along the line of sight, then the Faraday depth is, of course, reduced. Even if no thermal electrons are present at all, we may see Faraday rotation by the lowest energy relativistic electrons. The effective electron density to be inserted into Faraday depth calculations is $n_{\text {eff }} \sim n_{\text {rel }} / \gamma_{l}^{2}$, where $\gamma_{l}$ is the low-energy cutoff in the electron energy spectrum (Wardle 1977; Jones and O'Dell 1977). If the synchrotron radiation is produced by equal numbers of electrons and positrons, then $n_{\text {eff }}=0$.

Appreciable Faraday depth changes both the position angle and the degree of polarization (e.g., Burn 1966). Only in the case of a Faraday 'screen' external to and uniform over the angular scale of the source do we expect the familiar $\lambda^{2}$ rotation of position angle over more than a radian or so (Laing 1984). This gives external Faraday rotation a comparatively unambiguous signature. Internal Faraday rotation can exhibit more complex behavior, and for an inhomogeneous source it may not give a $\lambda^{2}$ rotation of position angle at all (Blandford and Königl 1979). If there is a spread of Faraday depths of more than a radian or so within an element of resolution, then the degree of polarization will be appreciably reduced ('front-back' depolarization in the case of internal Faraday rotation, or 'side-side' depolarization in either case; see, e.g., Conway et al. 1974).

It seems likely that there is not much Faraday rotation in most VLBI jets, although we wish to stress that this must be checked directly by simultaneous VLBI polarimetry at (at least) two wavelengths. Rudnick and Jones (1983) determined the rotation measures of 31 compact variable radio sources, using the VLA to separate extended ( $>$ arcsecond) structure. They found the resulting distribution of rotation measures to be indistinguishable from the distribution for extended sources, indicating that there is no additional nuclear component of rotation measure for these sources. Similar results have been found by Rusk (this symposium) and Wrobel et al. (this symposium). These measurements all include (or are made within) the $\lambda 18-20 \mathrm{~cm}$ band of the VLA. Therefore much of the polarized flux they detect is likely to come from components in the jet that either are further from the core than the components we observe or are resolved by us. Certainly, their rotation measures do not usually refer to the VLBI core itself, since this is often weakly polarized (see above). There is at least one source, 3C371 (Wrobel 1987), for which the 'VLA core' appears to have a much larger rotation measure $\left(380 \mathrm{rad} \mathrm{m}^{-2}\right)$ than a bright component near the core $\left(15 \mathrm{rad} \mathrm{m}^{-2}\right)$. We suspect there may be other cases of large core rotation measures which are discriminated against by the relatively long wavelength and low resolution of the VLA measurements.

Other evidence that the jet components usually have modest Faraday depths include the extremely high degree of polarization observed in $0 J 287$ (this rules out significant internal Faraday rotation), and the rough correlations found between the direction of the magnetic fields and the direction of the jets. These would not exist if these components had large excess rotation measures. Rudnick and Jones (1983) used these results to place interesting constraints on the nature of any plasma surrounding the jet.

The situation may be quite different for the VLBI cores. First, the integrated rotation measures discussed above may not be relevant. Second, while the jets coexist with the narrow-line emitting region (at least in galaxies and quasars), the cores are much closer to the broad-line emitting region, where densities are much higher (e.g., Ulrich 1984). Third, it is necessary to account for the very low degree of polarization observed in some of the cores $\left(m_{\text {core }}<1 \%\right.$ for $3 \mathrm{C} 120$ and $\left.3 \mathrm{C} 345\right)$. 
Optically thick synchrotron emission in a uniform magnetic field is about $10 \%$ polarized, so additional effects must be invoked. If the emitting region consists of $N$ cells, each with a random magnetic field direction, then the degree of polarization is reduced by a factor $\sim N^{-1 / 2}$. For an optically thick source, we would require $N \geq 100$. For an inhomogeneous source the requirements are more severe. A Blandford-Königl type jet is much more strongly polarized if the magnetic field is ordered $(m \sim 50 \%)$, because most of the jet is always optically thin (Cobb et al., this symposium). In this case we would require $N \geq 2500$. While this is possible, we consider it unlikely. The jets clearly contain regions of ordered magnetic field, and the high degrees of optical polarization imply ordered magnetic fields on a scale smaller than that of the VLBI core.

We therefore consider the possibility that the cores are depolarized by Faraday dispersion. This might be due to Faraday rotating material either inside the jet or surrounding the jet. Internal Faraday rotation is very inefficient at depolarizing a Blandford-Königl type jet, because the electron density and the magnetic field strength both decrease with distance down the jet, so the jet is always Faraday thin downstream. In terms of the Faraday depth at the $\tau=1$ surface, $\xi_{\tau=1}$, it can be shown that $m \propto \xi_{\tau=1}^{-1 / 4}$ (Cobb et al. this symposium). Thus enormous values for the Faraday depth are required to produce a net polarization as low as $1 \%$, and this is not compatible with the small Faraday depths observed in the jet components.

It therefore seems likely that the depolarization may occur outside the VLBI core, in the denser inner regions of the narrow-line emitting region. Physical parameters here are not well known, but if the magnetic field strength is not far below equipartition values, electron densities in the range $n_{e} \simeq 10^{5}-10^{7} \mathrm{~cm}^{-3}$ will provide the required Faraday depth. These densities are somewhat higher than are usually quoted for the narrow-line region in quasars and Seyfert I galaxies (e.g, Ferland 1981), but are consistent with more recent values found for the inner parts of this region (Fillipenko and Halpern 1984; De Robertis and Osterbrock 1984; Krolik and Vrtilek 1984).

Two pieces of circumstantial evidence are consistent with this picture. All the BL Lac objects that we have observed have appreciably more strongly polarized cores than $3 \mathrm{C} 120$ and 3C345. This is consistent with lack of a detectable emission line region in these objects. If observations of additional quasars and emission line galaxies show very weakly polarized VLBI cores, this would offer considerable support to this picture. In 3C345 we have observed new jet components, $\mathrm{C} 4$ and probably $\mathrm{C} 5$, emerging from the core (Wardle et al. this symposium). Their degrees of polarization are small, but appear to be increasing as their distance from the core increases, and their position angles of polarization differ from that of the bright knot $\mathrm{C} 3$ in the jet. This is also consistent with the picture of a Faraday thick screen surrounding the core. Indeed, it will be possible with VLBI polarimetry to map the radial distribution of Faraday depth in the nuclei of quasars and emission line galaxies by watching new components as they move away from the core. This will yield entirely new information on the narrow-line regions in these objects.

Financial support for this work was provided by the NSF under grants AST-82-13991 and AST-84-18636 (JFCW) and AST-83-15945 and AST-85-19529 (DHR).

\section{REFERENCES}

Aller, H. D., Aller, M. F., and Hughes, P. A. 1985, Ap. J., 298, 296.

Aller, H. D., Aller, M. F., Latimer, G. E., and Hodge, P. E. 1985, Ap. J. Suppl., 59, 513.

Altschuler D. R., and Wardle, J. F. C. 1976, Mem. R. A. S., 82, 1.

Altschuler D. R., and Wardle, J. F. C. 1977, M.N.R.A.S., 179, 153. 
Angel, J. R. P., and Stockman, H. S. 1980, Ann. Rev. Astr. Ap., 18, 321.

Båålh, L. B. 1984, in VLBI and Compact Radio Sources, ed. R. Fanti, K. Kellermann, and G. Setti (Dordrecht: Reidel), 127.

Begelman, M. C., Blandford, R. D., and Rees, M. J. 1984, Rev. Mod. Phys, 56, 255.

Biretta, J. A., Moore, R. L., and Cohen, M. H. 1986, Ap. J., 308, 93.

Björnsson, C.-I. 1982, Ap. J., 260, 855.

Blandford, R. D., and Königl, A. 1979, Ap. J., 232, 34.

Bridle, A. H., and Perley R. A. 1984, Ann. Rev. Astr. Ap., $22,319$.

Burn, B. J. 1966, M.N.R.A.S, 133, 67.

Carswell, R. F., Strittmatter, P. A., Williams, R. E., Kinman, T. D., and Serkowski, K. 1974, Ap. J. (Letters), 190, L101.

Chan, K. L., and Henriksen, R. N. 1980, Ap. J., 241, 534.

Cohen, M. H., Unwin, S. C., Pearson, T. J., Seielstad, G. A., Simon, R. S., Linfield, R. P., and Walker, R. C. 1983, Ap. J. (Letters), 269, L1.

Cohen, R. D., Smith, H. E., and Burbidge, E. M. 1986, B.A.A.S., 18, 674.

Conway, R. G., Haves, P., Kronberg, P. P., Stannard, D., and Wardle, J. F. C. 1974, M.N.R.A.S.,168, 137.

Cotton, W. D. et al. 1980, Ap. J. (Letters), 238, L123.

Cotton, W. D., Geldzahler, B. J., Marcaide, J. M., Shapiro, I. I., Sanromá, M., and Rius, A. $1984, A p$. J., 286, 503.

De Robertis, M. M., and Osterbrock, D. E. 1984, Ap.J., 286, 171.

De Young, D. S. 1986, Ap. J., 307, 62.

Ferland, G. J. 1981, Ap.J., 249, 17.

Filippenko, A. V., and Halpern, J. P. 1984, Ap.J., 285, 458.

Hughes, P. A., Aller, H. D., and Aller, M. F. 1985, Ap. J., 298, 301.

Jones, T. W., and O'Dell, S. L. 1977, Astron. Astrophys, 61, 291.

Jones, T. W., Rudnick, L., Aller, H. D., Aller, M. F., Hodge, P. E., and Fiedler, R. L. 1985, Ap.J., 290, 627.

Königl, A., and Choudhari, A. R. 1985, Ap.J., 289, 173.

Krolik, J. H., and Vrtilek, J. M. 1984, Ap.J., 279, 521.

Laing, R. A. 1980, M.N.R.A.S, 193, 427.

Laing, R. A. 1984, in Physics of Energy Transport in Extragalactic Radio Sources., NRAO Workshop No. 9, ed. A. H. Bridle and J. A. Eilek (Greenbank, WV: National Radio Astronomy Observatory), 90.

Ledden, J. E., and Aller, H. D. 1978, in Pittsburgh Conference on BL Lac Objects, ed. A. M. Wolfe (Pittsburgh: University of Pittsburgh), 60.

Miller, J. S., French, H. B., and Hawley, S. A. 1978, in Pittsburgh Conference on BL Lac Objects, ed. A. M. Wolfe (Pittsburgh: University of Pittsburgh), 176.

Moore, R. L., et al. 1982, Ap. J., 260, 415.

Moore, R. L., Schmidt, G. D., and West, S. C. 1987, Ap. J., 314, 176.

Mutel, R. L., and Phillips, R. B. 1984, in VLBI and Compact Radio Sources, ed. R. Fanti, K. Kellermann, and G. Setti (Dordrecht: Reidel), 117.

Pauliny-Toth, I. I. K., Porcas, R. W., Zensus, A., and Kellermann, K. I. 1984, in VLBI and Compact Radio Sources, ed. R. Fanti, K. Kellermann, and G. Setti (Dordrecht: Reidel), 149.

Roberts, D. H., Gabuzda, D. C., and Wardle, J. F. C. 1987, Ap. J., submitted.

Roberts, D. H., Potash, R. I., Wardle, J. F. C., Rogers, A. E. E., and Burke, B. F. 1984, in VLBI and Compact Radio Sources, ed. R. Fanti, K. Kellermann, and G. Setti (Dordrecht: Reidel), 35.

Roberts, D. H., and Wardle, J. F. C. 1986, in Quasars, ed. G. Swarup and V. K. Kapahi, 
(Dordrecht: Reidel), 141.

Roberts, D. H., and Wardle, J. F. C. 1987, in Superluminal Radio Sources, ed. J. A. Zensus and T. J. Pearson, (Cambridge: Cambridge University Press), 193.

Rudnick, L., and Jones, T. W. 1983, A. J., 88, 518.

Rudnick, L., Jones, T. W., Edgar, B. K., and Pedalty, J. A. 1984, A. J., 89, 316.

Rusk, R., and Seaquist, E. R. 1985, A. J., 90, 30.

Simard-Normandin, M., Kronberg, P. P., and Button, S. 1981, Ap. J. Suppl., 45, 97.

Sitko, M. L., Schmidt, G. D., and Stein, W. A. 1985, Ap. J. Suppl., 59, 323.

Sitko, M. L., and Junkkarinen, V. T. 1985, P.A.S.P., 97, 1158.

Smith, P. S., Balonek, T. J., Elston, R., and Heckert, P. A. 1987, Ap. J. Suppl., 64, 459.

Stockman, H. S., Angel, J. R. P., and Miley, G. K. 1979, Ap. J. (Letts.), 227, L55.

Ulrich, M.-H. 1984, in VLBI and Compact Radio Sources, ed. R. Fanti, K. Kellermann, and G. Setti (Dordrecht: Reidel), 73.

Walker, R. C., Benson, J. M., and Unwin, S. C. 1987, Ap. J., 316, 546.

Wardle, J. F. C. 1977, Nature (London), 269, 563.

Wardle, J. F. C., Roberts, D. H., Potash, R. I., and Rogers, A. E. E. 1986, Ap. J. (Letters), 304, L1.

Wardle, J. F. C., and Roberts, D. H. 1986a, Can. J. Phys., 64, 434.

Wardle, J. F. C., and Roberts, D. H. 1986b, in Continuum Emission in Active Galactic Nuclei, Proceedings of KPNO Workshop, ed. M. L. Sitko, (Tucson: National Optical Astronomy Observatories), 190.

Wolfe, A. M., Davis, M. M., and Briggs, F. H. 1982, Ap. J., 259, 495.

Wrobel, J. M. 1987, in Superluminal Radio Sources, ed. J. A. Zensus and T. J. Pearson, (Cambridge: Cambridge University Press), 186. 